\title{
A Reply to Breznau, Rinke, and Wuttke on the Discordant Outcomes of Hypothesis Testing
}

\author{
Daniel Goldman
}

October 2021

\section{Introduction}

In April 2021, Breznau, Rinke, Wuttke, et al. published a preprint concerning an exercise to test the consistency of hypothesis testing across multiple researchers, using the same data set and hypothesis[1]. The test revealed that even with the same hypothesis and the same data set, there was a diverse array out outcomes, including non-significant, negative, and positive outcomes. It may seem baffling that there could be such discord between hypothesis tests. And that's because no such thing exists.

The issue at hand is something other than a failure to reach the same conclusion using the same hypothesis and the same data. The problem is that different hypotheses are being used, without awareness, and thus different conclusions are made. The reason for this failure is that scientists are often too sloppy with the construction of their hypothesis tests in the first place, and this sloppiness arises from a misunderstanding of how science works. When testing a hypothesis, there is only one correct test which is exact. Any other test is a mere approximation of the hypothesis test which coincides with a given hypothesis. To understand why, we must understand how scientific inquiry functions. And once we do, we can solve many of the problems found in the aforementioned research.

\subsection{The Findings}

The paper in question has some interesting, and rather disturbing conclusions. The study analyzed the results of 73 teams. They supposedly tested the same hypothesis with the same data. Even though there were only 73 research groups, there were 107 research decisions. According to the paper, $13.5 \%$ stated that the hypothesis "was not testable." Another $60.7 \%$ stated that the hypothesis should be rejected. And $28.5 \%$ stated that the hypothesis was supported. The variation in outcomes was poorly explained by factors such as differences in expertise, and even researcher bias. Most of the variation was simply explainable, based on the observations made. Indeed, the authors concluded that "the massive variation 
in reported results originated from unique or idiosyncratic decisions in the data analysis process, leaving $95.2 \%$ of the total variance in results unexplained."

\section{The Nature of Scientific Inquiry}

The final statement alone shows an error in the academic understanding of scientific inquiry. There is no such thing as supporting the hypothesis. Hypothesis testing is a statistical form of proof by contradiction, and the nature of scientific inquiry is one of falsification, not verification[2]. We learn this reality very early on. But many are not taught why there is no verification in science. But even then, very early in our education, we learn that we cannot accept the null hypothesis: that a failure to reject it is never an acceptance of it. Why? The answer is quite straight forward. The hypothesis test does one thing and one thing only. It assumes that a given null hypothesis is true, outputs the probability of an observation occurring, under that assumption, and compares that probability a threshold for rejection.

Essentially, we assume $H_{0}$ AND $p_{o}<p_{t}$ where $p_{o}$ is the probability of our observation and $p_{o}$ is our threshold. This threshold is somewhat arbitrary, unfortunately, but it can be made more rigorous by analyzing how much existing data and testing is already done on the theory which generated the hypothesis. From this combined assumption, we can see that a contradiction in the assumption merely leads to the conclusion that either $H_{0}$ is false or that we were too conservative in our threshold. Another important point to realize is that the null hypothesis is more than just a single equality or inequality. The null hypothesis is a statement about the probability distribution of expected outcomes of a given experiment. An experiment itself is simply a set of independent variables, their states, and one dependent variable which is to be measured. Because the null hypothesis includes not just the equality or inequality that we normally describe, but also includes the distribution itself, the statistical test that must be chosen is already fixed. And when we reject the null hypothesis, it could still be that our target value is correct, and that the distribution was simply wrong.

But where does the hypothesis itself come from? For instance, if we have a collection of data S, how do we know what the mean of that data should be? How do we know if we can assume that the data was pulled from a normal distribution or not? We can test for normality, but why should we even do so? I've seen many cases where hypothesis tests and other analysis tools are just thrown around randomly, without any care about what the data is supposed to represent. I've seen linear regression done on a data set which clearly should not have been linear. The problem is that people do not seem to understand, even still, that science is a system of falsification, and that we therefore start with a theory that we are testing, unless we are simply engaging in exploratory data collection and analysis. Therefore, the theory itself will tell you what the hypothesis is, and thus what the test should be. Therefore when obtain a contradiction, it is a contradiction against the theory that generated the hypothesis.

For instance, a person's height is a function of numerous random events 
summed together. The theory of biological growth demands that if sampled properly, heights should be normally distributed. However, if the data points were measures of wealth in a population, then we cannot make such assumptions, as wealth distribution, based on our current theories, is not normally distributed. If two researchers are getting different results for the same data, then they are not using the same hypothesis test, or at the very least are relying on two different thresholds for the cut-off. But if a researcher is using a different test on the same data for the same theory, then at least one has made a mistake. And they may incorrectly reject or fail to reject the null hypothesis and make an incorrect evaluation of the theory, as they are relying on logical implications which did not arise from assuming that the theory is true! The one caveat here is if the actual test is too complicated and it is being estimated. But even then the estimate is for the true hypothesis which is already determined by the theory.

Unfortunately, it is very difficult to formalize a theory to the point where it is absolutely certain which hypothesis is generated for a given experiment. We rely on a high degree of subjectivity at this point. However, we can still improve the situation so that we do not obtain such disparate results. We can begin to establish better protocols for arguing why a given hypothesis, and thus hypothesis test, is implied by the theory being tested. This protocol of course requires that we become more accustomed to clearly describing the theory that we intend to test. A rigorous deductive process from theory to hypothesis test will ensure that we have more uniform results. It will also allow us to better check for errors in the deductive steps.

\section{Conclusion}

It is important that scientists understand how science works in order to properly conduct scientific research. Some variation in hypothesis testing results may be due to factors such as relying on different approximations of the actual hypothesis test that must be performed. And some can be explained away by using different thresholds of rejection. However, the ultimate realization that comes from the results of Breznau, Rinke, Wuttke, et al. is that researchers are ignoring the nature of scientific inquiry itself. They are treating it as an inductive system that constructs and confirms theories. They are not rigorously defining the parameters of their theories and hypotheses, before starting their tests. Science is not a hypothesis test. Science is the process through which we provisionally eliminate false assumptions. These assumptions are our theories. They may come from past experiences or not. But once a theory is selected for testing, everything else follows. And until scientists take the time to fully understand the philosophy of science, the kind of phenomenon discussed by Breznau, Rinke, Wuttke, et al. will likely continue to be a major problem. 


\section{References}

[1] Nate Breznau et al. "Observing Many Researchers Using the Same Data and Hypothesis Reveals a Hidden Universe of Uncertainty". In: (Mar. 2021). DOI: 10.31222/osf.io/cd5j9. URL: https://doi.org/10.31222/osf . io/cd5j9.

[2] Daniel S Goldman. "The Basics of Hypothesis Tests and Their Interpretations". In: (Aug. 2018). DOI: 10 .31219/osf . io/u2csn. URL: https : //doi.org/10.31219/osf.io/u2csn. 